\title{
Editorials
}

Alexander J. Shysh MD FRCPC, Chris J. Eagle MD FRCPC FANZCA

\section{The characteristics of excellent clinical teachers}

ly rated teachers better or merely more popular? Is there any objective outcome evidence that these "good" teachers were any more effective than their more moderately rated peers?

The results of the study must be questioned, but they appear to be consistent with the information available in the educational and medical literature concerning the four components of effective teaching identified by the authors. These components are the characteristics of good teachers, the proper use of teaching methods, the use of case based learning, and the importance of inquiry.

Characteristics of good teachers have been studied widely in both the educational ${ }^{5-7}$ and medical education $^{8-11}$ literature. In fact, these characteristics are similar to those previously described for good clinical teachers in anaesthesia. ${ }^{12}$ These include such strengths as an interest in teaching, willingness to engage in discussion, content knowledge, good interpersonal and communication skills, competent performance in clinical care, intellectual honesty, the ability to show residents respect, and flexibility and cordiality while teaching. Enthusiasm and willingness to teach appear to be most important factors in promoting student learning in the operating room. ${ }^{b}$ The authors of the current article have confirmed these attributes through their ethnographic study.

The authors have identified a process of teaching in the operating room consistent with a wide body of literature. Educational researchers have described a presage-process-product model of the daily instructional events that delineate the pre-instruction, during instruction, and post-instruction tasks for teaching. ${ }^{5,13-14}$ The instructional events as outlined by the authors parallel these educational models and mirror the instructional events for operating room teaching as described by Eagle, such as first recognizing the learner's level of development or training, setting expectations or objectives for the learning encounter, questioning the learner about the clinical case in a problem-solving fashion, using evaluative questions to 
probe the learner's comprehension and to give direction to the teaching, and finally providing a summary and appropriate feedback to the learner. ${ }^{15}$

Similarly, the authors' recognition of the importance of case-based teaching has support from medical education, especially its processes and associated benefits. ${ }^{16,17}$ Furthermore, the authors' finding of the importance of proper constructive feedback techniques has been recognized in the field of medical education. . $^{18,19}$

Finally, there is support for the effectiveness of inquiry and apprenticeship models. In particular, the cognitive apprenticeship model of teaching and learning $^{20}$ has been found to have application in orthopaedic ${ }^{21}$ and anaesthesia ${ }^{a}$ education.

The study is interesting in that it concentrates on the developmental level of the teacher and his or her philosophy towards education, rather than merely their teaching skills. Thus, it goes beyond all other investigations into the characteristics of excellent anaesthesia teachers. However, teaching effectiveness may be enhanced also by identifying the developmental level of the student. ${ }^{22,23}$ Recently, the teacher's teaching style or method has been seen to need careful matching to the student's level of self directed learning in order to enhance learning outcome. ${ }^{24,25}$ Perhaps the good teachers identified in this study were better able to teach uncertain or complex topics effectively to more senior residents. In contrast, most teachers may be effective in an authority or expert teaching mode with junior and more dependent learners.

From their interviews with teachers, the authors identify other issues of concern. Teaching the skills required to give an acceptable anaesthetic is a critical component of our training programmes, but it is not our only responsibility. The Royal College of Physicians and Surgeons of Canada Accreditation Standards ${ }^{\mathrm{b}}$ and the recently developed CanMEDS 2000 Project $^{\mathfrak{C}}$ emphasize the professional characteristics of our trainees and graduates. Issues such as medical ethics, communication, and attitude are of increasing importance. If even our best teachers feel unprepared to deal with these issues directly, then there is reason for concern and impetus for change. Our programmes need the ability to train our teachers to adapt to the changing environment. A well planned system of faculty development is increasingly important.

"How to make time" is a constant concern for clinical teachers. Frequently unfunded and with a wide diversity of demands, clinical teachers risk frustration and burnout. Given the initiation of health care reform in many regions of Canada, financial stress coupled with increasing administrative and clinical responsibilities will only increase the demands on our clinical teachers. This must be a major issue for academic departments in the short term and all anaesthetists in the long run.

While the research performed by Cleave-Hogg and Benedict ${ }^{4}$ was qualitative, the results are consistent with work from other areas of education. The study has at least construct validity. Do the authors prove their hypothesis? The authors state that a better "educational climate" is one that promotes greater complexity of thinking, which can only be achieved if teachers have a "holistic view of teaching and learning." It is impossible with this type of study to make the bridges from teacher characteristics to educational process to improved outcome of learning. The authors have shown that "better" teachers appear to function at high epistemological levels. Improving our knowledge of the characteristics of effective teachers is a necessary part of developing strategies to enhance the learning of our students.

In order to improve rationally the process of clinical education in the operating room, we need to know much more about the process of learning, the characteristics of the teacher, and the learning ability of the student in that environment. This study is provocative because it exposes our lack of understanding and the limited amount of work done in this area.

What are the characteristics of excellent clinical teachers in anaesthesia? This question has perplexed many and the results remain unclear. While several common characteristics have been delineated for excellent clinical teachers, those who attempt to exhibit such characteristics frequently become frustrated since they are not always viewed as being effective teachers by their students. There appears to be more to the excellent clinical teacher than one who is enthusiastic, willing, and dedicated to teaching. In response, Cleave-Hogg and Benedict, in examining another dimension of the excellent clinical teacher, have gone beyond these commonly accepted attributes. ${ }^{4}$ They have opened the door to further research by looking at the teacher's level of philosophical development towards teaching. This track will probably be one of many different ones taken when attempting to define comprehensively the excellent clinical teacher.

\section{Footnotes}

a Shysh, AJ. The nature of anaesthesia education in the operating room at The University of Calgary (thesis). Calgary, Canada: University of Calgary, 1996. 175p.

b General Standards of Accreditation. The Royal College of Physicians and Surgeons of Canada. Ottawa, Canada; 1994.

c Societal Needs Working Group, CanMEDS 2000 Project. Skills for the New Millennium. Ann R Coll Physicians Surg Can 1996; 29: 206-16. 


\section{Les caractéristiques de l'excellence en éducation}

Plusieurs anesthésistes remplissent une tâche importante : l'enseignement clinique, Ils sont toutefois mal formés et mal orientés pour l'accomplir. C'est au pifomètre qu'ils apprennent à enseigner et ils ne peuvent compter que sur un feed-back sporadique pour s'améliorer. ${ }^{a}$ Ce rôle revêt tout de même une importance capitale. L'enseignement bien fait assure l'avenir de la qualité des soins, procure de meilleures connaissances aux étudiants et attire les meilleurs candidats. $\grave{A}$ cause de ce manque de formation spécifique, le petit nombre de publications en rapport avec l'enseignement ayant subi le jugement des pairs n'a rien de surprenant. Étant donné son importance et un manque évident de ressources, nous devons donc redoubler nos efforts pour améliorer au maximum l'enseignement clinique.

Dans les années 1970, Paget s'était permis quelques observations sur l'enseignement en salle d'opération mais elles n'avaient pas eu de suites tangibles. ${ }^{1-3}$ Nous ne possédons pas de critères permettant de distinguer l'enseignement de qualité de l'enseignement médiocre issu des meilleures intentions. Même si les anesthésistes participent à des cours de perfectionnement sur l'enseignement offerts par des associations professionnelles comme la Society for Education in Aneasthesia, il n'en demeure pas moins, qu'à la base, l'information provient de l'expérience personnelle ou de l'extrapolation de l'expérience des autres disciplines. En salle d'opération, la qualité de l'enseignement ne repose pas sur des exigences déterminées scientifiquement. La recherche de la qualité conduira éventuellement à la réalisation d'études observationnelles et expérimentales. Dans ce numéro, CleaveHogg et Benedict présentent le compte rendu d'une étude ethnographique conçue pour déterminer les caractéristiques des bons professeurs cliniques et leur façon de participer au développement de la connaissance (l'épistémologie). ${ }^{4}$

Les problèmes que nous éprouvons à interpréter les résultats de leur étude résident dans la méthode utilisée. Les auteurs n'ont identifié que les caractéristiques qu'elles recherchaient chez les «meilleurs» parmi les professeurs, mais les professeurs "médiocres» se seraient-ils comportés différemment ! Auraient-ils révélé un développement épistémologique du même degré ? Auraient-ils employé des méthodes péda- gogiques différentes ? Les professeurs grandement appréciés sont-ils meilleurs ou simplement plus populaires ? Existe-t-il des preuves objectives qui démontrent que ces «bons" professeurs sont plus efficaces que leurs homologues moins bien cotés?

On peut se poser bien des questions sur leurs résultats mais ceux-ci semblent en accord avec ce que la littérature médicale et pédagogique nous transmet sur les quatre volets de l'enseignement efficace identifiés par les auteures: ce qui distingue les bons professeurs, des méthodes d'enseignement appropriées, l'orientation vers les soins et l'usage du questionnement.

Les caractéristiques des bons professeurs ont été largement étudiées dans la littérature pédagogique ${ }^{5-7}$ et médicale. ${ }^{8-11}$ En réalité, ce sont les mêmes qui s'appliquent aux professeurs cliniques d'anesthésie déjà décrites. ${ }^{12}$ Elles comprennent des points forts comme l'intérêt pour l'enseignement, la volonté d'engager la discussion, la connaissance du contenu, l'habileté à communiquer, la compétence clinique, l'honnêteté intellectuelle, la considération envers le résident, la cordialité et la flexibilité des rapports pendant l'enseignement. L'enthousiasme et l'attrait pour l'enseignement semblent les facteurs les plus importants pour l'avancement de l'enseignement en salle d'opération. ${ }^{a}$ Les auteures du présent article ont confirmé ces attributs dans cette étude ethnographique.

La description du processus d'enseignement en salle d'opération que nous font les auteures est conforme à celui d'une grande partie de la littérature. Les spécialistes en éducation ont décrit pour l'acte pédagogique quotidien un modèle intuition-méthode-résultat qui délimite pour l'enseignant les tâches qui s'appliquent aux phases qui précèdent, accompagnent et suivent la formation. ${ }^{5,13-14}$ Les actes pédagogiques résumés par les auteures correspondent à ces modèles et reflètent l'activité pédagogique spécifique à la salle d'opération telle que décrite par Eagle, comme la constatation préalable du degré de développement ou de formation de l'élève, le questionnement de l'élève dirigé de façon à résoudre un cas problème, l'utilisation de questions normatives et un feed-back pertinent. ${ }^{15}$

De la même façon, les observations des auteures spécialement en ce qui concerne les méthodes et les avantages de l'apprentissage par problèmes sont conformes à celles des éducateurs médicaux, ${ }^{16,17}$ de même que l'importance des techniques constructives de feed-back. ${ }^{18,19}$

Finalement, l'efficacité des modèles d'apprentissage avec questionnement de l'étudiant a fait des adeptes. En particulier, l'orthopédie ${ }^{21}$ et l'anesthésie ont adopté ce modèle d'apprentissage pour l'enseignement et l'acquisition des connaissances. 
Cette étude est intéressante parce qu'elle est centrée sur le degré de développement du professeur et sur sa philosophie face à l'éducation plutôt que sur ses habiletés pédagogiques. Elle va ainsi plus loin que toutes les recherches qui ont été réalisées sur les caractéristiques de l'enseignant d'excellente qualité. Cependant l'efficacité de l'enseignement peut aussi être rehaussée par l'identification du niveau de développement de l'étudiant. ${ }^{22,23}$ Il est à noter que, depuis peu, on réalise la nécessité pour l'enseignant d'harmoniser sa méthode ou son style avec le niveau de l'auto-apprentissage de l'étudiant afin d'obtenir de meilleurs résultats. ${ }^{24,25}$ Dans cette étude, les «bons» professeurs étaient peut-être plus aptes à enseigner des sujets obscurs ou compliqués à des résidents plus avancés. Par contre, avec des résidents juniors et plus dépendants, il est possible que la plupart des professeurs réussissent mieux avec un type d'enseignement magistral ou autoritaire.

Les entrevues avec les professeurs auront permis aux auteures de cerner d'autres préoccupations. L'enseignement des compétences nécessaires à l'administration de l'anesthésie fait partie intégrale de nos programmes d'enseignement, mais il n'est pas notre seule responsabilité. Les Normes générales d'agrément du Collège royal des médecins et des chirurgiens $d u$ Canada ${ }^{\mathrm{b}}$ et le projet CanMEDS $2000^{\mathrm{C}}$ mettent l'accent sur les caractéristiques professionnelles de nos étudiants et de nos diplômés. Des sujets comme l'éthique, les communications, et les attitudes prennent de plus en plus d'importance. Si même nos meilleurs professeurs se sentent mal préparés à affronter ces problèmes directement, nous devons nous inquiéter et inciter aux changements. Nos programmes doivent être conçus pour aider nos professeurs à s'adapter à un milieu en pleine évolution. II devient donc très important de d'élaborer un plan de développement pour le corps professoral.

Les professeurs cliniques se demandent constamment s'ils auront suffisamment de temps pour enseigner. Souvent sous-financés et sollicités de toutes parts, ils risquent la frustration et l'épuisement. À cause des nouvelles réformes des soins de santé en vigueur dans plusieurs régions du Canada, les contraintes financières associées aux responsabilités administratives et cliniques croissantes ne feront qu'intensifier les pressions sur nos professeurs cliniques. Ceci constitue un problème majeur à court terme pour les départements universitaires et à long terme pour l'ensemble des anesthésistes.

Même si la recherche de Cleave-Hogg et de Benedict $^{4}$ portait sur la qualité, ses résultats sont cohérents avec des travaux exécutés dans d'autres domaines de l'éducation. Cette étude est pour le moins valide dans sa conception. Les auteures ontelles prouvé leur hypothèse ? Les auteures énoncent que, pour l'éducation, un meilleur climat favorise une plus grande complexité intellectuelle qui ne peut être atteinte qu'avec «une vision holistique de l'enseignement et de l'apprentissage» de la part des professeurs. Il est impossible avec ce type d'étude de faire le lien entre les caractéristiques de l'enseignant, le processus pédagogique et le succès de l'enseignement. Les auteures ont montré que les meilleurs enseignants semblaient fonctionner à un niveau épistémologique plus élevé. L'amélioration de nos connaissances des caractéristiques du bon professeur constitue une partie essentielle de nos stratégies destinées à renforcer la formation de nos étudiants.

Pour améliorer le processus de l'enseignement clinique en salle d'opération, il faut, dans ce même environnement, mieux comprendre le processus d'acquisition des connaissances, les caractéristiques du professeur et l'habileté de l'étudiant à apprendre. Cette étude dérange parce qu'elle illustre bien notre manque de compréhension et le peu de travail réalisé dans ce domaine.

Quelles sont les caractéristiques de l'enseignant clinique de qualité en anesthésie ? Cette question en a déconcerté plus d'un et les réponses demeurent vagues. Alors que plusieurs caractéristiques communes aux enseignants cliniques de qualité ont été cernées, ceux qui tentent d'y adhérer sont souvent frustrés parce qu'ils ne sont pas reconnus comme tels par leurs étudiants. Il semble que la volonté, l'enthousiasme et dévouement ne suffisent pas. En réponse, en étudiant une autre dimension de l'enseignement de qualité, Cleave-Hogg et Benedict sont allées encore plus loin que les attributs généralement reconnus. ${ }^{4}$ Elles ont ouvert la porte à une recherche ultérieure en propicau développement d'une philosophie de l'enseignement. Cette voie sera vraisemblablement une parmi plusieurs empruntées pour la définition globale de l'enseignant clinique de qualité.

\section{Footnotes}

a Shysh, AJ. The nature of anaesthesia education in the operating room at The University of Calgary (Thèse). Calgary, Canada: Université de Calgary, 1996. 175p.

b Normes générales de l'agrément. Le Collège royal des médecins et chirurgiend du Canada. Ottawa, Canada; 1994.

c Socital Needs Working Group, CanMEDS 2000 Project. Skills for the New Millenium. Ann R Coll Physicians Surg Can 1996; 29: 206-16. 


\section{References}

1 Paget NS, Lambert TF. Tutor-student interaction in the operating theatre. Anaesth Intensive Care 1976; 4 : 301-3.

2 Lambert TF, Paget NS. Teaching and learning in the operating theatre. Anaesth Intensive Care 1976; 4: 304-7.

3 Paget NS, Eaton E. Teaching anaesthetics in the operating room. Anaesth Intensive Care 1977; 5: 247-50.

4 Cleape-Hogg D, Benedict C. Characteristics of good anaesthesia teachers. Can J Anaesth 1997; 44: 587-591.

5 Rosenshine B, Stevens R. Teaching functions. In: Whittrock MC (Ed.). Handbook of Research on Teaching, 3rd ed. New York: Macmillan Publish Co., 1986: 376-91.

6 Power B. Instructor Excellence. Mastering the Delivery of Training. San Francisco: Jossey-Bass Publishers, 1992.

7 Sternberg RJ, Horvath JA. A prototype view of expert teaching. Educational Researcher 1995; 24: 9-17.

8 McLeod PJ, Hardin RM. Clinical teaching strategies for physicians. Medical Teacher 1985; 7: 173-89.

9 Harth SC, Bavanandan S, Thomas KE, Lai MY, Thong $\Upsilon H$. The quality of student-tutor interactions in the clinical learning environment. Med Educ 1992; 26 : 321-6.

10 Price DA, Mitchell CA. A model for clinical teaching and learning. Med Educ 1993; 27: 62-8.

11 Irby $D M$. Teaching and learning in ambulatory care settings: a thematic review of the literature. Acad Med 1995; 70: 898-931.

12 Rhoton MF, Cascorbi HF. Good clinical teachers: what do/don't they do? Anesthesiology 1985; 63: A491.

13 Clark CM, Peterson PL. Teachers' thought processes. In: Whittrock MC (Ed.). Handbook of Research on Teaching, 3rd ed. New York: Macmillan Publishing Co., 1986: 225-96.

14 Biggs JB, Telfer $R$. The Process of Learning, 2 nd ed. Sydney, Australia: Prentice-Hall, 1987.

15 Eagle C. Anaesthesia and education. Can J Anaesth 1992; 39: 158-65.

16 Kroenke $K$. Attending rounds: guidelines for teaching on the wards. J Gen Int Med 1992; 7: 68-75.

17 Irby DM. Three exemplary models of case-based teaching. Acad Med 1994; 69: 947-53.

18 Ende J. Feedback in clinical medical education. JAMA 1983; 250: 777-81.

19 Brookfield S. Giving helpful evaluation to learners. Adult Learning 1992; 3: 22-4.

20 Collins A, Brown JS, Newman SE. Cognitive apprenticeship: teaching the crafts of reading, writing, and mathematics. In: Resnick LB (Ed.). Knowing,
Learning and Instruction. Essays in honor of Robert Glaser. New Jersey: Lawrence Erlbaum Associates, 1989: 453-94.

21 Farmer JA Jr, Lippert FG III, Schafer MF. Making orthopaedic education interactive, problem oriented, and on target. In: Eilert RE (Ed.). Instructional Course Lectures. Park Ridge, Illinois: American Academy of Orthopaedic Surgeons, 1992: 481-6.

22 Benner P. From novice to expert. Am J Nursing 1982; 82: 402-7.

23 Shuell TJ. Phases of meaningful learning. Review of Educational Research 1990; 60: 531-47.

24 Pratt $D D$. Andragogy as a relational construct. Adult Education Quarterly 1988; 38: 160-81.

25 Grow GO. Teaching learners to be self-directed. Adult Education Quarterly 1991; 41: 125-49. 